\title{
Actinobaculum shaalii: a new uropathogen?
}

\author{
Valentina Felice, Massimiliano Scutellà, Silvia Lombardi \\ Analysis Laboratory, A. Cardarelli Hospital, Regione Molise Health Unit, Campobasso, Italy
}

\section{Summary}

Background and Aims. Actinobaculum schaalii is a facultative anaerobic, Gram-positive rod-shaped species phylogenetically related to Actinomyces. A. schaalii is an emerging pathogen causing urinary tract infections (UTI) in both children and adults; although, as part of the human genitourinary tract flora, it is frequently overlooked or considered as a contaminant. While the phenotypic identification of $A$. schaalii is difficult, the recent Matrix-Assisted Laser Desorption/Ionisation Time-Of-Flight-mass spectrometry (MALDI TOF) technology could represent a promising tool for its identification.

Materials and Methods. This is a retrospective study including all known cases $(\mathrm{n}=7)$ of $A$. schaalii infections occurred (between July 2013 and November 2013) at the Microbiology Laboratory of the A. Cardarelli Hospital, in Campobasso (Italy).

Results. All the 7 A. schaalii collected strains, resulted in vitro susceptible to most of the drugs commonly used for urinary tract infections, but resistant to ciprofloxacin, a first-line antibiotic in the treatment of prostatitis. All isolates were susceptible to amoxicillin, amoxicillin-clavulanic, ampicillin-sulbactam, cefuroxime, gentamicin, piperacillin-tazobactam, vancomicin, tetracycline (no EUCAST breakpoints). All except two isolates were susceptible to cefotaxime; $3 / 7$ and $5 / 7$ strains were clindamicin and levofloxacin resistant, respectively.

Conclusions. As most antibiotics empirically prescribed for UTI (mainly fluoroquinolones or trimethoprim/sulfamethoxazole) are not

\footnotetext{
Correspondence: Massimiliano Scutellà, Laboratorio Analisi, P.0. A. Cardarelli, Contrada Tappino, 86100 Campobasso, Italy

Tel.:+39.0874.409375.

E-mail: maxscutella@hotmail.it
}

Key words: Actinobaculum shaalii; urinary tract infection; antimicrobial susceptibility.

Contributions: the authors contributed equally.

Conflict of interest: the authors declare no potential conflict of interest.

Received for publication: 28 October 2014.

Accepted for publication: 18 December 2014.

CCopyright V. Felice et al., 2015

Licensee PAGEPress, Italy

Microbiologia Medica 2015; 30:4801

doi:10.4081/mm.2015.4801

This article is distributed under the terms of the Creative Commons Attribution Noncommercial License (by-nc 3.0) which permits any noncommercial use, distribution, and reproduction in any medium, provided the original author(s) and source are credited. effective against $A$. schaalii, the appropriate onset of treatment was delayed by an average of 2.8 days. The implementation of the newer MALDI TOF technology in routine diagnostic procedures may allow a more reliable and rapid identification of $A$. schaalii in future.

\section{Introduction}

Actinobaculum schaalii was first described in 1997 and named after Klaus P. Schaalii, a German microbiologist specialized in Actinomicete microbiology. The genus Actinobaculum includes A. schaalii (1997), A. suis (1997), A. massiliense (2002) and A. urinale (2003) and is closely related to the genera Actinomyces and Arcanobacterium (12).

A. schaalii is a small Gram-positive, non-motile, asporigenous, facultative anaerobic, coccoid rod that requires $\mathrm{CO}_{2}$ for optimal growth (Figure 1).

Hippurate is hydrolyzed whereas esculin (except a few exceptions) and gelatin are not (Table 1). Relatively few sugars are fermented (i.e. glucose, maltose, ribose, and D-xylose). The isolates produce $\alpha$-glucosidase, alanine-phenylalanine-proline arylamidase, and pyroglutamic acid arylamidase whereas alkaline phosphatase, arginine dihydrolase, $\alpha$-galactosidase, $\beta$-galactosidase, $\beta$-glucuronidase, and $\beta$-mannosidase activities are not detected. They do not reduce nitrates to nitrites, and acetoin is not produced $(7,12,15,16,20)$.

It grows slowly after $48 \mathrm{~h}$ in anaerobic atmosphere or microaerophilic condition at $37^{\circ} \mathrm{C}$ as tiny grey colonies, less than 1 $\mathrm{mm}$ in diameter, and shows weak $\beta$-hemolysis on agar plates containing 5\% horse or sheep blood after 3-5 days of growth $(13,20)$ (Figure 2). It is catalase, oxidase and urease negative and resistant to trimethoprim and ciprofloxacin (15). It is easily overgrown by other bacteria, which are often found concomitantly. Because of its slow anaerobic growth and resemblance to the normal bacterial flora on skin and mucosa, A. schaalii is often overlooked or considered a contaminant. These difficulties regarding detection and identification prevent the evaluation of the clinical impact of this pathogen and of its potential to cause invasive infection.

Most laboratories do not look specifically for Actinobaculum species. Even in the case of $A$. schaalii growth, this microorganism may be overlooked either because of overgrowth of other common uro-pathogens or due to the bacteria's similarity to normal skin flora $(3,15,19)$.

A. schaalii has been reported to be responsible for UTIs, mainly in elderly patients with underlying urological risk factors $(2,7,13,15)$. Interestingly, a recent study showed that $22 \%$ of 252 urine samples from patients $>60$ years were positive for $A$. schaalii using real-time PCR. This microorganism may also cause septic complications such as urosepsis, abscess, osteomyelitis and endocarditis $(2,9,10,13$, $15,14,18)$. It has also been recovered from other human clinical specimens than urine such as blood, but its pathogenic potential remains unknown. Although A. schaalii is an emerging uro-pathogen, in vitro antimicrobial agents susceptibilities have been poorly evaluated, particularly in terms of MICs. 
Difficulties identifying $A$. schaalii by using traditional phenotypic tests have obscured its pathologic role for many years. Our laboratory recently introduced the Bruker MALDI-TOF identification system which uses the Biotyper database. Given the ease and rapidity in species identification, this technology is now used to screen all blood culture samples (to evaluate the pure/predominant bacterial growth both from sterile and non sterile sites). Indeed, mass spectra $(\mathrm{m} / \mathrm{z}$ range between 2000 and 13000) obtained for A. schaalii strains appear to be unique and reproducible. Notingly, all the databases now available do not include the spectrum profile for this species and must be updated $(11,17)$.

The aim of this study was i) to define the demographic and clinical characteristics of all the patients affected by an A. schaalii infection, ii) to identify the particular elements enabling clinicians to suspect an $A$. schaalii infection and consequently to request its specific detection.

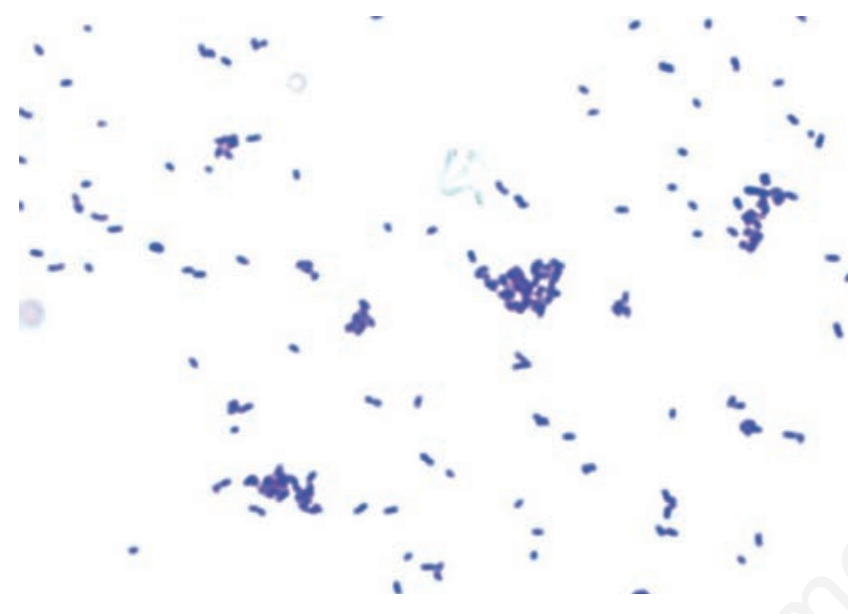

Figure 1. Gram stain of Actinobaculum shaalii.

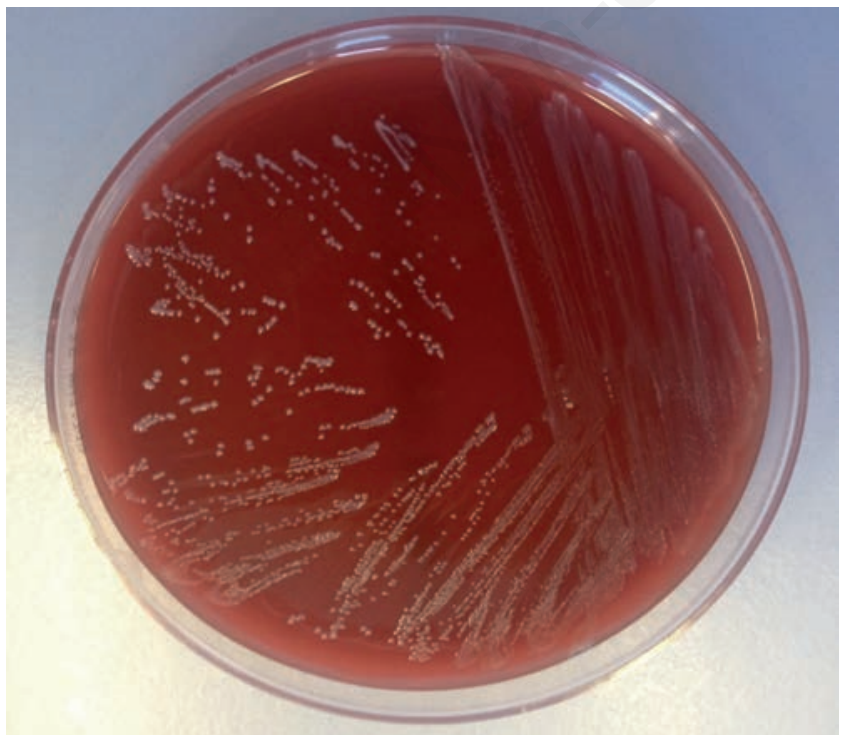

Figure 2. Colonies morphology of Actinobaculum shaalii grown on Columbia agar medium with $5 \%$ sheep blood after $48 \mathrm{~h}$ of anaerobic incubation at $37^{\circ} \mathrm{C}$.

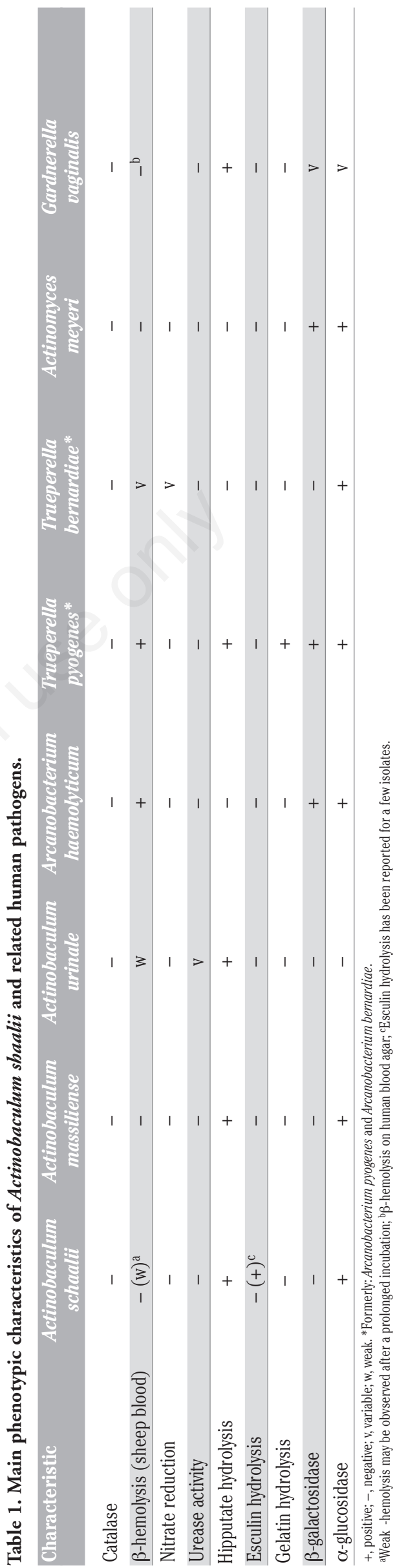




\section{Materials and Methods}

\section{Patients and data collection}

This is a retrospective study including all known cases of $A$. schaalii infections (from July to November 2013) at the Microbiology Laboratory of Cardarelli Hospital in Campobasso. All the data on the patients, specimens and consecutive non-replicate isolates of $A$. schaalii, were obtained from the database of the Clinical Microbiology Laboratory.

Only the cases from patients with genitourinary site infections caused by $A$. schaalii were analyzed. An infectious diseases specialist reviewed the medical records of all the patients: patient's age and sex, underlying diseases or health condition, clinical signs at the time of detection of $A$. schaalii, administered antibiotic therapy, and clinical outcome. The antibiotic regimen chosen after the identification of the pathogen was considered as those related to the outcome. The cases were categorized as colonization or infection.

\section{Culture and species identification of Actinobaculum schaalii}

We examined seven clinical isolates of A. schaalii recovered from urine, urethral swab and seminal fluid.

Grey, tiny colonies with weak $\beta$-hemolysis or without hemolysis on $5 \%$ Columbia sheep blood agar after $48 \mathrm{~h}$ of anaerobic (or $5 \% \mathrm{CO}_{2}$ ) incubation were further analyzed.

All isolates showing Gram-positive coccoid rods and a negative catalase reaction were identified by MALDI TOF system (Bruker-Daltonics) with a score of about 2.3 (i.e., a reliable score to the species level) using the direct colony transfer method $(5,6,11)$ (Figure 3 ). All bacteria growing as monocultures in normally sterile specimens and collected from unsterile body sites were considered to be clinically relevant.

\section{Antimicrobial susceptibility testing}

Even though no recommendations have been established yet to perform antimicrobial susceptibility testing (AST) for Actinobaculum schaalii, the E-test method seems to be reliable $(1,2,4,8,9,15)$.

MICs of the following 12 antibiotics were determined for all strains using E-test (Liofilchem): Amoxicillin, amoxicillin-clavulanic, ampicillin-sulbactam, piperacillin-tazobactam, cefotaxime, cefuroxime,

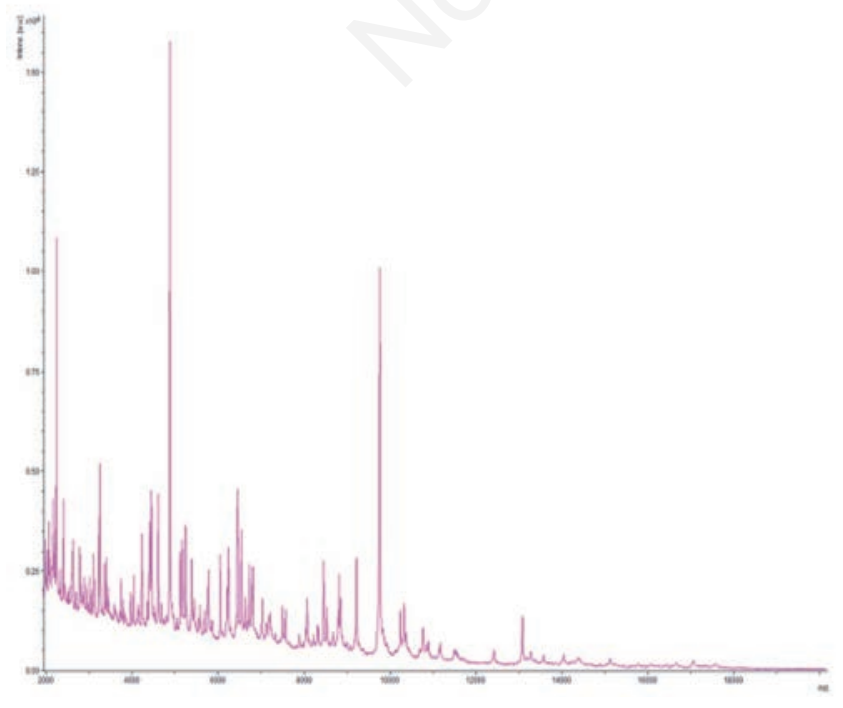

Figure 3. Mass spectra of Actinobaculum schaalii.

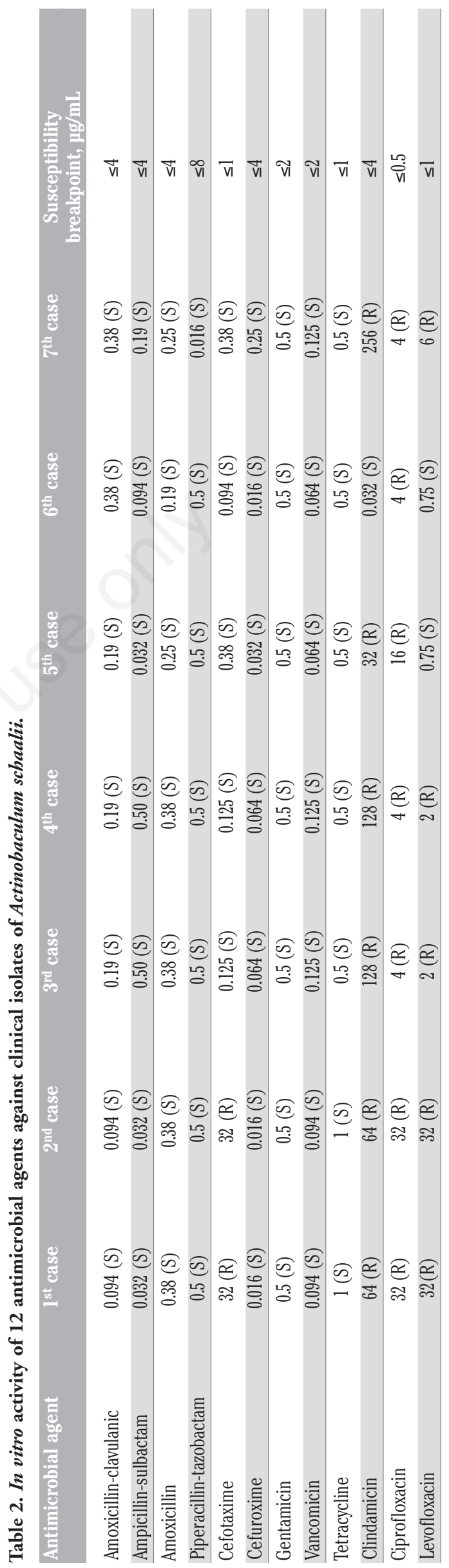


ciprofloxacin, levofloxacin, clindamycin, gentamicin, vancomicin, tetracycline.

In vitro susceptibilities were performed on Mueller-Hinton blood agar in $5 \% \mathrm{CO}_{2}$ using an inoculum of $0.5 \mathrm{McFarland}$. The plates were incubated $48 \mathrm{~h}$ in anaerobic conditions. Bacteroides fragilis ATCC 25285 was used as a quality control. No clinical breakpoints have been recommended yet for A. schaalii, but interpretation of MIC results may be done using non-species-related interpretative standards or those established for the other uro-pathogens (Table 2).

\section{Results}

Between July 2013 and November 2013 we identified 7 A. schaalii positive samples in 7 male patients. Specimens positive for $A$. schaalii were found in urine, urethral swab and seminal fluid. All the patients were symptomatic for an UTI, showing urinary symptoms such as alguria and dysuria. All specimens resulted mono-microbic. The patient's ages ranged from 24 to 39 years (mean 30). All patients were not hospitalized.

The isolates were identified as Actinobaculum schaalii by MALDI TOF mass spectrometry, with a score from 2.15 to 2.36 (mean 2.25) using the direct colony transfer method.

Being informed on the prevalence of the causative UTIs pathogens (and their antimicrobial susceptibility) in different patient's types is of great importance to make a correct clinical and microbiological diagnosis. The A. schaalii isolates studied, in vitro susceptible to most of the drugs commonly used for UTIs, resulted resistant to ciprofloxacin, a first-line antibiotic in prostatitis treatment. All the isolates were susceptible to amoxicillin, amoxicillin-clavulanic, ampicillin-sulbactam, cefuroxime, gentamicin, piperacillin-tazobactam, vancomicin, tetracycline (EUCAST breakpoints). Five out of seven isolates were susceptible to cefotaxime, $3 / 7$ and 5/7 were resistant to clindamicin and levofloxacin, respectively. As most antibiotics empirically prescribed for urinary infection (mainly fluoroquinolones or trimethoprim/sulfamethoxazole) are not effective against $A$. schaalii, the appropriate onset of treatment was delayed by an average of 2.8 days (range 1-13).

\section{Discussion}

UTIs are predominantly caused by members of the Enterobacteriaceae family. However, Gram-positive bacteria and polymicrobial infections are not uncommon in patients with underlying urological conditions. $A$. schaalii is an underestimated opportunistic uro-pathogen and its search is relevant in cases of unexplained chronic pyuria, especially when there is discrepancy between smear microscopy findings and growth results under aerobic conditions $(13,15)$.

Although A. schaalii is an emerging uro-pathogen, very little information are available about its in vitro antibiotics susceptibility, especially to those commonly used in UTIs treatment (e.g. ciprofloxacin). In addition slow growing bacteria such as $A$. schaalii can remain undetected for the overgrown of faster-growing Enterobacteria. Implementation of the newer MALDI TOF technology in routine diagnostic procedures may allow a more rapid identification of $A$. schaalii, which might increase the frequency of its detection.

A. schaalii has traditionally been thought to be part of the commensal flora of the human urogenital tract (12). Colonization seems to be a risk factor for an $A$. schaalii UTI both in children and adults, as most UTI case reports have been described in the same age groups in which colonization has been most frequently detected.

The vast majority of $A$. schaalii isolates were resistant to ciplofloxacin, frequently used as empiric treatment in UTIs in children and adults. Current recommendations for the antibiotic treatment for A. schaalii infections are represented by amoxicillin or cephalosporins. Data suggesting that gentamicin, vancomicin, linezolid, nitrofurantoin and meticillinam are potential alternative treatment options are limited $(1,4)$. The optimal length period of antibiotic therapy in the case of an $A$. schaalii infection is currently unknown. Most case reports suggest a treatment duration of 7 to 14 days.

Therefore we repeated the urine culture after eight days of antibiotic treatment, and stopped treatment after 14 days as soon as negative culture results were available.

\section{Conclusions}

In conclusion, $A$. schaalii is an emerging pathogen in both children and adults. Colonization and subsequent infection seem to be influenced by a number of factors, in particular age of the patient. The spectrum of infections extends from benign cystitis to pyelonephritis with bacteriemia, indicating that the pathogen can be invasive. A. schaalii is likely to have been under-diagnosed in the past, but with introduction of the MALDI TOF system, a rapid and inexpensive technology, $A$. schaalii should specifically be included in the microbiological search of pathogens instead of dismissing its presence as clinically irrelevant colonization by Corynebacteria or Lactobacillus spp.

Microbiology laboratory management include selective blood agar plates and identification by MALDI-TOF System.

A prospective study on $A$. schaalii infections should be performed to confirm these results and determine the appropriate treatment and its duration.

\section{References}

1. Andersen PK, Soby KM, Bank S, Prag J. In vitro susceptibility of Actinobaculum schaalii to mecillinam. J Antimicrob Chemother 2011; 66: 2181-2.

2. Bank S, Jensen A, Hansen TM, et al. Actinobaculum schaalii, a common uropathogen in elderly patients, Denmark. Emerg Infect Dis 2010; 16: 76-80.

3. Beguelin C, Genne D, Vara A, et al. Actinobaculum schaalii: clinical observation of 20 cases. Clin Microbiol Infect 2011; 17: 1027-11.

4. Cattoir V, Varca A, Greub G, et al. In vitro susceptibility of Actinobaculum schaalii to 12 antimicrobial agents and molecular analysis of fluoroquinolone resistance. J Antimicrob Chemother 2010; 65: 2514-7.

5. Dusch H, Burren K, Hinrikson HP. 25 July 2010, posting date. MALDI-TOF mass spectrometry for routine identification of medically relevant bacteria. Unilabs Mittelland, Berne, Switzerland.

6. Farfour E, Leto J, Barritault M, Barberis C, et al. Evaluation of the Andromas matrix-assisted laser desorption ionization-time of flight mass spectrometry system for identification of aerobically growing Gram-positive bacilli. J Clin Microbiol 2012; 50: 2702-7.

7. Fendukly F, Osterman B. Isolation of Actinobaculum schaalii and Actinobaculum urinale from a patient with chronic renal failure. $J$ Clin Microbiol 2005; 43: 3567-9.

8. Gomez E, Gustafson DR, Rosenblatt JE, Patel R. Actinobaculum bacteremia: a report of 12 cases. J Clin Microbiol 2011; 49: 4311-3.

9. Haller P, Bruderer T, Schaeren S, et al. Vertebral osteomyelitis caused by Actinobaculum schaalii: a difficult-to-diagnose and potentially invasive uropathogen. Eur J Clin Microbiol Infect Dis 2007; 26 : 667-70. 
10. Hoenigl M, Leitner E, Valentin T, et al. Endocarditis caused by Actinobaculum schaalii, Austria. Emerg Infect Dis 2010; 16: 1171-3.

11. La Scola B, Fournier PE, Raoult D. Burden of emerging anaerobes in the MALDI-TOF and $16 \mathrm{~S}$ rRNA gene sequencing era. Anaerobe 2011; 17: 106-12.

12. Lawson PA, Falsen E, Akervall E, et al. Characterization of some Actinomyces-like isolates from human clinical specimens: reclassification of Actinomyces suis (Soltys and Spratling) as Actinobaculum suis comb. nov. and description of Actinobaculum schaalii sp. nov. Int J Syst Bacteriol 1997; 47: 899-903.

13. Nielsen HL, Soby KM, Christensen JJ, Prag J. Actinobaculum schaalii: a common cause of urinary tract infection in the elderly population. bacteriological and clinical characteristics. Scand J Infect Dis 2010; 42: 43-7.

14. Pajkrt D, Simoons-Smit AM, Savelkoul PH, et al. Pyelonephritis caused by Actinobaculum schaalii in a child with pyeloureteral junction obstruction. Eur J Clin Microbiol Infect Dis 2003; 22: 438-40.
15. Reinhard M, Prag J, Kemp M, et al. Ten cases of Actinobaculum schaalii infection: clinical relevance, bacterial identification, and antibiotic susceptibility. J Clin Microbiol 2005; 43:5305-8.

16. Sarkonen N, Kononen E, Summanen P, et al. Phenotypic identification of Actinomyces and related species isolated from human sources. J Clin Microbiol 2001; 39: 3955-61.

17. Stingu CS, Rodloff AC, Jentsch H, et al. Rapid identification of oral anaerobic bacteria cultivated from subgingival biofilm by MALDITOF-MS. Oral Microbiol Immunol 2008; 23: 372-6.

18. Sturm PD, Van EJ, Veltman S, et al. Urosepsis with Actinobaculum schaalii and Aerococcus urinae. J Clin Microbiol 2006; 44: 652-4.

19. Tschudin-Sutter S, Frei R, Weisser M, et al. Actinobaculum schaalii-invasive pathogen or innocent bystander? A retrospective observational study. BMC Infects Dis 2011; 11: e289.

20. Vanden Bempt I, Van Trappen S, Cleenwerck I, et al. Actinobaculum schaalii causing Fournier's gangrene. J Clin Microbiol 2011; 49: 2369-71. 